Notes \& Tips

\title{
Biosensor plates detect mitochondrial physiological regulators and mutations in vivo
}

\author{
Anabela P. Rolo a, Carlos M. Palmeira a , Gino A. Cortopassi ${ }^{\mathrm{b}, *}$ \\ ${ }^{a}$ Center for Neurosciences and Cell Biology, Department of Zoology, University of Coimbra, 3004-517 Coimbra, Portugal \\ b VM: Molecular Biosciences, University of California at Davis, 1 Shields Avenue, Davis, CA 95616, USA
}

\section{A R T I C L E I N F O}

\section{Article history:}

Received 15 September 2008

Available online 7 October 2008

\begin{abstract}
A B S T R A C T
The measurement of mitochondrial activity in living cells is usually not straightforward, even though it is quite important in physiological and pathophysiological processes. We describe a high-throughput method for measurement of mitochondrial oxygen consumption in living cells, based on the BectonDickinson Biosensor plates.
\end{abstract}

(C) 2008 Elsevier Inc. All rights reserved.
Mitochondria are the primary energy-generating system in most eukaryotic cells. As the site of oxidative phosphorylation, these double-membrane organelles provide a highly efficient route for eukaryotic cells to generate ATP from energy-rich molecules. Classic mitochondrial disorders result from mutations in mtDNA or nuclear genes that disrupt mitochondrial respiratory function [1]. Additionally, several neurodegenerative diseases also result in mitochondrial defects. Thus, analysis of mitochondrial dysfunction is of particular importance in drug development and the evaluation of selective mitochondrial targeting of therapeutic molecules.

Despite the specific and informative nature of oxygen consumption as an indicator of mitochondrial and cellular function, it is not a widely used parameter owing to difficulties in its evaluation in cells in culture using traditional polarographic methods. Isolation of mitochondria from cells in culture usually damages them, producing respiratory control ratios of 2 or less. Mitochondrial oxygen consumption and ATP synthesis are also measured in digitoninpermeabilized tissue culture cells using traditional polarographic methods. However, this assay has limitations related with the high number of cells necessary and limited sample throughput.

The recent use of quenched-fluorescence oxygen sensing is an extremely simple, broadly applicable noninvasive assay that rapidly provides informative insights into oxygen consumption in cell culture models [2-6]. The intensity and lifetime of the emitted fluorescence vary inversely with oxygen concentration. Oxygensensing probes have been shown to be a simple, high-throughput means to evaluate oxygen consumption in isolated mitochondria in vitro [5,6], with the results favorably compared to polarographic analysis in vitro.

\footnotetext{
Corresponding author. Fax: +1 5307549342.

E-mail address: gcortopassi@ucdavis.edu (G.A. Cortopassi).
}

The Becton-Dickinson Biosensor plates were originally described in 2000 [2] and have been used mostly to monitor cell proliferation and to perform cytotoxicity and viability assays [2-4]. The plates consist of an oxygen-sensitive fluorophore (tris-4,7diphenyl-1,10-phenanthroline ruthenium(II) chloride) in a silicone rubber matrix in the bottom of each well of a standard microplate. The high-throughput format (96 and 384 wells) enables the repeated and noninvasive monitoring of cellular oxygen consumption completely in an in vivo situation. In cultured cells, this biosensor system has been used to determine $\mathrm{O}_{2}$ consumption as an indication of the state of a cell culture [4], as well as an indication of functional viability of islets [7], but not mitochondrial physiology. A limitation of the assay is its relatively low sensitivity, because mitochondria in vivo are not maximally stimulated unless perturbed, i.e., by an increase in [ADP] or a loss in membrane potential.

Thus, we tried to improve the sensitivity of the plates by adding the mitochondrial uncoupling agent p-trifluoromethoxy carbonyl cyanide phenyl hydrazone (FCCP) and thus evaluating the maximal rate of oxygen consumption. We also explored how many parameters of mitochondrial function could be demonstrated in vivo and whether point mutations, deletions, and ablation of the mitochondrial genome affected oxygen consumption by the assay, as well as drugs known to affect mitochondrial proliferation.

\section{Materials and methods}

We cultured 143B osteosarcoma cells, 143B cells depleted of mtDNA (rho-zero), and 143B cells bearing either the 11778 LHON point mutation (for Leber's hereditary optic neuropathy) or the 4977 mtDNA deletion in Dulbecco's modified Eagle's medium (DMEM; Invitrogen, Carlsbad, CA, USA) supplemented with $10 \%$ fetal bovine serum, $100 \mathrm{mM}$ sodium pyruvate, $5 \mathrm{mg} / \mathrm{ml}$ uridine, 
and $50 \mathrm{mg} / \mathrm{ml}$ gentamycin. The 143B cells were kindly provided by M. King, rho-zero cells were kindly provided by M. Zeviani, 143B cells with the 11778 mutation and fusion controls were kindly provided by A. Martinuzzi, and 143B cells bearing the 4977 deletion were kindly provided by $\mathrm{C}$. Moraes.

Cells were harvested by trypsinization and washed with PBS. The whole cell pellets were then suspended in DMEM and $0.2 \times 10^{6}$ cells were added to individual wells of a 96-well BD Oxygen Biosensor System plate (BD Biosciences, San Jose, CA, USA), followed by DMEM to a final volume of $200 \mu \mathrm{l}$ per well. The plates were then maintained for $30 \mathrm{~min}$ at $37^{\circ} \mathrm{C}$ in a humidified incubator with $5 \%$ $\mathrm{CO}_{2}$. For drug treatments, compound stock solutions were prepared in dimethyl sulfoxide and added to the wells to give the indicated final concentrations. Plates were scanned in a temperature-controlled $\left(37^{\circ} \mathrm{C}\right.$ ) plate reader (Cytofluor; PE Biosystems, Foster City, CA, USA) with an excitation wavelength of $485 \mathrm{~nm}$ and an emission wavelength of $630 \mathrm{~nm}$. Measurements were taken $1 \mathrm{~min}$ apart for a total of $40 \mathrm{~min}$ and the time profiles of fluorescence intensity in each well were analyzed using Excel software (Microsoft). The fluorescence traces in each well were normalized to the signal in air-saturated buffer. Slopes of fluorescence signal were calculated in the dynamic range of the measurements to compare respiratory rates of different samples. We also evaluated whether, owing to the incomplete sample sealing in the microplate formats and the associated back diffusion of atmospheric oxygen, the measured rates of dissolved oxygen using this approach were lower than the consumption rates measured using a sealed system and tried sealing with gel, plastic wrap, and foil. However, sealed and unsealed plates produced the same general trends in FCCP-stimulated oxygen consumption (data not shown).

To confirm that the measured fluorescence varies inversely with oxygen concentration, and to determine the dynamic range of the assay, sodium sulfite was used as a positive control to eliminate all molecular oxygen. Sodium sulfite increased the fluorescent signal to $\approx 20,000$ relative fluorescence units ( $R F U$ ), defining the maximal possible response and confirming the $\mathrm{O}_{2}$ dependence.

Total DNA was extracted from untreated and pioglitazone- or rosiglitazone-treated cells using a ZR Genomic DNA Kit (Zymo Research, Orange, CA, USA). DNA concentration was determined by spectrophotometry. PCRs were performed with a Roche LightCycler (Roche Diagnostic, Indianapolis, IN, USA) according to a method previously described [8].

\section{Results and discussion}

The chemical uncoupler FCCP stimulated oxygen consumption reproducibly in 143B cells in a dose-dependent fashion (Fig. 1A).
Rates of oxygen consumption were calculated from the slope of the fluorescence curve from 0 to $20 \mathrm{~min}$. One, 2.5 , and $5 \mu \mathrm{M} \mathrm{FCCP}$ stimulated mean oxygen consumption 1.1-, 2.5-, and 7.4-fold over the basal rate. Five micromolar FCCP produced the maximal stimulation of oxygen consumption. Thus in living cells a 7-fold stimulation of mitochondrial electron flow by FCCP is possible, greatly increasing the sensitivity of the biosensor plates.

If the stimulation of oxygen consumption was the result of FCCP-uncoupled respiration and not some extramitochondrial function, inhibiting the mitochondrial electron transport chain (ETC) should block it. The ETC inhibitor cyanide completely abolished oxygen consumption, demonstrating that mitochondria are the major source of oxygen consumption in the cell (Fig. 1A). Rotenone, an inhibitor of complex I, added after FCCP, blocked the increase in $\mathrm{O}_{2}$ consumption, but FCCP added after rotenone could not increase $\mathrm{O}_{2}$ consumption, as expected (Fig. 1B). Antimycin a and stigmatellin, inhibitors of complex III, and 3-nitropropionic acid, an inhibitor of complex II, produced the same inhibition of $\mathrm{O}_{2}$ consumption as rotenone (data not shown). Although NADH oxidation by complex I is the main source of electrons to the ETC, the inhibition of succinate dehydrogenase by 3-nitropropionic acid blocks the tricarboxylic acid (TCA) cycle and consequently reduces NADH availability to electron transport. The addition of all electron transport chain inhibitors caused not only a flattening of slope but a slight reversal, which is likely explained by "requenching," i.e., rebinding of $\mathrm{O}_{2}$ dissolved in the medium back to the probe (Fig. 1B).

As shown in Fig. 1B, oligomycin, a specific inhibitor of the mitochondrial $F_{0} F_{1}$-ATP synthase, caused a reduction in $\mathrm{O}_{2}$ consumption compared to untreated cells, demonstrating coupling of $\mathrm{O}_{2}$ consumption to ATP synthesis in the living cell by the assay.

Thapsigargin, which causes release of calcium from endoplasmic reticulum (ER) stores, produced an increase in $\mathrm{O}_{2}$ consumption that was inhibitable by the mitochondrial calcium uniporter inhibitor ruthenium red and also by cyanide (data not shown). These data are explained by the fact that calcium must enter mitochondria through the uniporter in order to decrease the membrane potential and stimulate mitochondrial electron flow.

Point mutations, deletions, and decreases in the mitochondrial genome cause Leber's hereditary optic neuropathy, chronic progressive external ophthalmoplegia (CPEO), and mitochondrial depletion syndrome, respectively, and several other mitochondrial diseases [9]. The 143B cells bearing the LHON 11778 mutation, a CPEO heteroplasmic 7.5-kb deletion each, had about half the rate of oxygen consumption of the controls, and the rho-zero cells had essentially zero oxygen consumption (Fig. 2A).
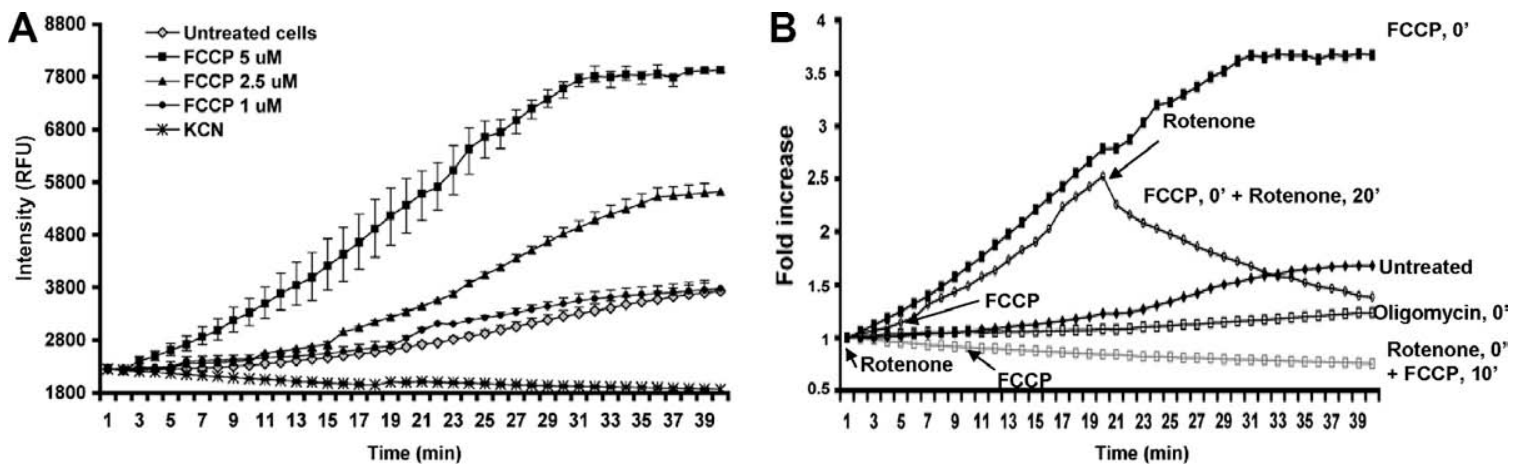

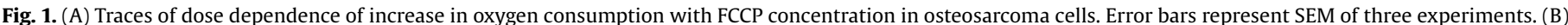

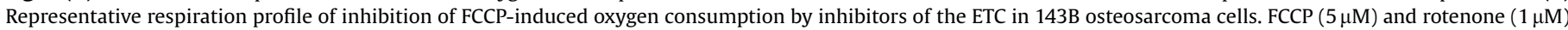

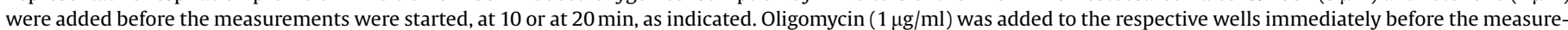
ments were started. Data are presented as fold increase of initial intensity. 

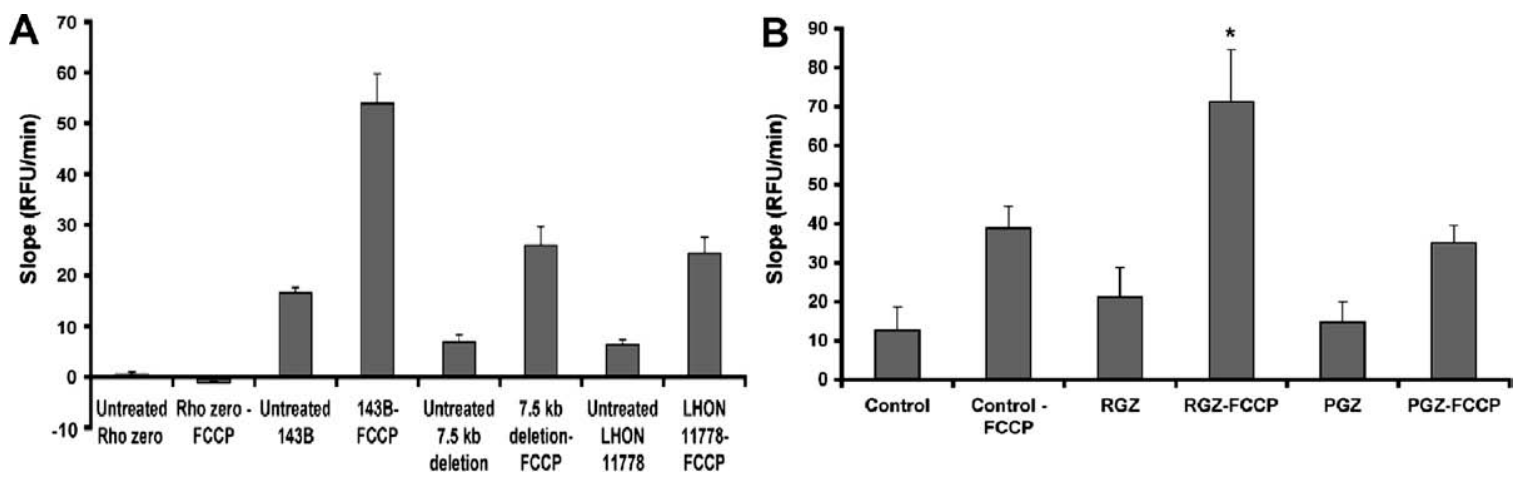

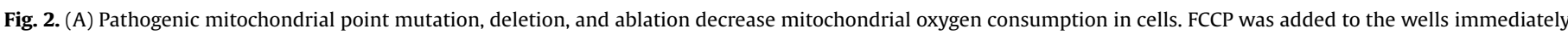

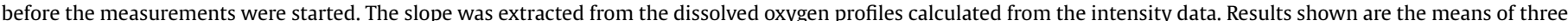

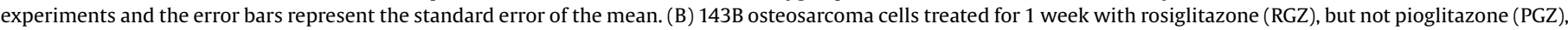

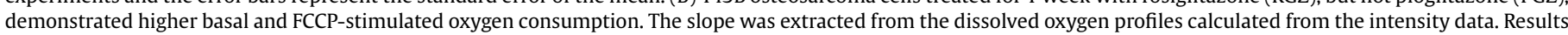

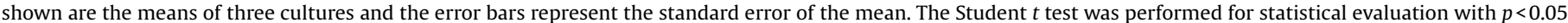
considered to be significant $(*)$.

One reasonable application of a high-throughput, plate-based assay for mitochondrial oxygen consumption is to try to identify drugs that modify this process. One way to increase mitochondrial capacity is through amplification or inhibition of mitochondrial copy number in the cell. Since rosiglitazone and pioglitazone have been demonstrated to increase mitochondrial copy number [10], 143B cells, 143B cells with the 11778 mutation. and 143B cells bearing the 4977 deletion were treated for 1 week with $10 \mu \mathrm{M}$ pioglitazone or $10 \mu \mathrm{M}$ rosiglitazone. The mean FCCP-stimulated rate of oxygen consumption was 1.4-fold higher in cells treated with rosiglitazone (Fig. 2B). The increased rates were explainable by increased mitochondrial copy number, which was significantly increased $(170 \%)$ in rosiglitazone-treated cells; pioglitazone produced a trend for increase (140\%), which was not statistically significant (data not shown).

Another possible way to stimulate mitochondrial performance is to increase the maximal rate of electron transfer through the ETC. Two drugs that are currently in clinical use to treat mitochondrial diseases, including CPEO and LHON, are coenzyme Q and its more bioavailable counterpart, idebenone. We observed no increase in $\mathrm{O}_{2}$ consumption in 143B cells treated with $50 \mu \mathrm{M}$ coenzyme $\mathrm{Q}$ or with 1,10 , or $50 \mu \mathrm{M}$ idebenone for $30 \mathrm{~min}$ (data not shown).

In summary, we have improved a high-throughput assay for $\mathrm{O}_{2}$ consumption, using it to show in living cells in vivo that (1) nearly all cellular $\mathrm{O}_{2}$ consumption is mitochondrial, (2) point mutations and deletions of the mtDNA genome decrease $\mathrm{O}_{2}$ consumption, (3) stimulation of ER calcium release stimulates mitochondrial $\mathrm{O}_{2}$ consumption, (4) inhibition of complex II blocks all mitochondrial $\mathrm{O}_{2}$ consumption, and (5) rosiglitazone produces increased mitochondrial oxygen consumption consistent with increased copy number in osteosarcoma cells.

The measurement of maximal oxygen consumption by FCCP treatment of cells on BD Biosensor plates is a sensitive, fast, and specific screening method for drugs or other small molecules that enhance mitochondrial oxygen consumption in cell mod- els of human pathologies. We believe that the improved assay described here could help to identify novel, potentially therapeutic, compounds that affect mitochondrial copy number or electron flow, in a high-throughput manner.

\section{Acknowledgments}

A.P. Rolo is the recipient of a fellowship from the Fundação para a Ciência e a Tecnologia (SFRH/BPD/26514/2006), and this work was supported by grants from the USPHS (EY12245, AG11967, AG16719, and AG23311 to G.A.C.).

\section{References}

[1] S. DiMauro, E.A. Schon, Mitochondrial respiratory-chain diseases, N. Engl. J. Med. 348 (2003) 2656-2668.

[2] M. Wodnicka, R.D. Guarino, J.J. Hemperly, M.R. Timmins, D. Stitt, J.B. Pitner Novel fluorescent technology platform for high throughput cytotoxicity and proliferation assays, J. Biomol. Screen. 5 (2000) 141-152.

[3] J. Hynes, S. Floyd, A.E. Soini, R. O'Connor, D.B. Papkovsky, Fluorescence-based cell viability screening assays using water-soluble oxygen probes, J. Biomol Screen. 8 (2003) 264-272.

[4] R.D. Guarino, L.E. Dike, T.A. Haq, J.A. Rowley, J.B. Pitner, M.R. Timmins, Method for determining oxygen consumption rates of static cultures from microplate measurements of pericellular dissolved oxygen concentration, Biotechnol. Bioeng. 86 (2004) 775-787.

[5] J. Hynes, L.D. Marroquin, V.I. Ogurtsov, K.N. Christiansen, G.J. Stevens, D.B. Papkovsky, Y. Will, Investigation of drug-induced mitochondrial toxicity using fluorescence-based oxygen-sensitive probes, Toxicol. Sci. 92 (2006) 186-200.

[6] Y. Will, J. Hynes, V.I. Ogurtsov, D.B. Papkovsky, Analysis of mitochondrial func tion using phosphorescent oxygen-sensitive probes, Nat. Protocol. 1 (2006) 2563-2572.

[7] W. Wang, L. Upshaw, D.M. Strong, R.P. Robertson, J. Reems, Increased oxygen consumption rates in response to high glucose detected by a novel oxygen biosensor system in non-human primate and human islets, J. Endocrinol. 185 (2005) 445-455.

[8] A. Wong, G. Cortopassi, Reproducible quantitative PCR of mitochondrial and nuclear DNA copy number using the LightCycler, Methods Mol, Biology 197 (2002) 129-137.

[9] S. Dimauro, G. Davidzon, Mitochondrial DNA and disease, Ann. Med. 37 (2005) $222-232$. 